\title{
Research on Control Method of Turning Plate Based on Image Processing Technology
}

\author{
Chunyu $\mathrm{He}^{1, \text { a }}$, Zhijie Jiao ${ }^{1, b}$, Xiaogang Wu, Jun Wang ${ }^{1, \mathrm{~d}}$ \\ ${ }^{1}$ State Key Lab of Rolling and Automation, Northeastern University, Shenyang 110819, China

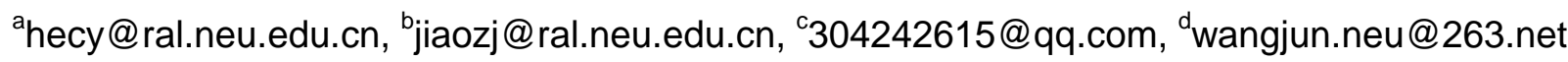

Keywords: image process, plate, automatic turning plate, angle measurement

Abstract. In this paper, the image characteristics of rectangular parts like plate analyzed in this paper in view of the demand for automatic truning plate in the rolling process. Image processing technology is used to identify the image, based on the edge detection algorithm, contour tracking algorithm and edge detection algorithm to achieve the size and angle of the rectangular parts of the plate. Based on the construction of the plate image detection and measurement system, the automatic turning plate control algorithm is developed, which realizes the automatic production process control of the plate plant, and meets the requirements of the process to produce rhythm control, and improves the production efficiency.

\section{Introduction}

In plate production process, in order to get arbitrary plate width by several fixed size of slab production, the plate will be rotated 90 degrees in some pass, namely turning plate operations. The turning plate is realized through the tapered roller, as shown in Fig. 1, odd and even roller arranged alternately, the odd roller is divided into a group, even roll into another group, were independently driving. When the odd roller and even roller speed in the same direction, the plate is in forward or reverse transport state, when the odd roller and even roller speed in the opposite direction, the plate in turning state.

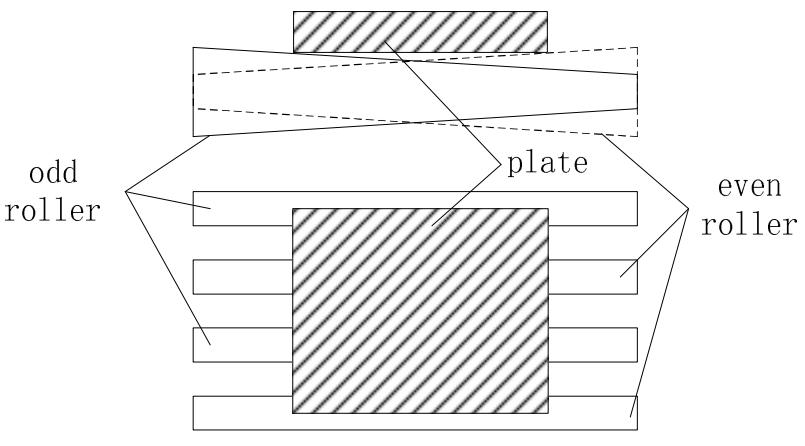

Fig. 1 The composition of rotation table

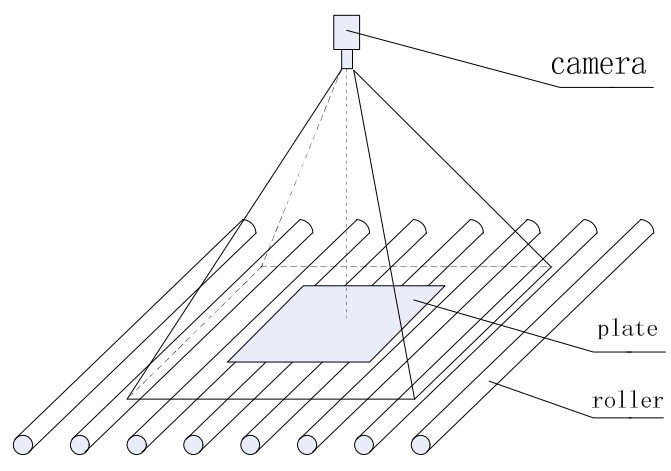

Fig. 2 Installation way of camera

According to the mathematical model of the system, the automatic rolling system of plate realizes the scheduling and control to production process, the operation process is basically without manual intervention $[1,2]$. At present, the only missing part of the automatic rolling system, which needs to manually intervene, is the function of turning plate. In the turn plate pass, the operator need switch from the automatic rolling way to manual mode. After the manual turning plate, the rolling state needs to be confirmed to resume to the automatic rolling. The artificial interference in the process of turning plate is the disturbance of the automatic rolling system, which affects the production process of the high grade product and is not conducive to system stability. Automatic turning plate method can make the plate production process to achieve the true sense of the automatic rolling, without human intervention, improve the production efficiency, so that the production process and rhythm control with high reproducibility and accuracy. 


\section{Plate angle measurement and control}

Turning plate control is usually arranged in the first several passes. At this time the slab temperature is higher (above 950). Using the thermal radiation of the slab itself, the image is collected by CCD camera installed in the top of the turning plate roller, digital image can be input to the computer via image acquisition card, as the plate position identification object [3-5]. CCD camera installation method is shown in Fig. 2.

The plate angle is calculated by detecting the image edge. After using the image processing algorithm to get the plate edge image, the boundary tracking algorithm is used to get the boundary points set. The specific plate angle measuring method is as follows:

The Image preprocessing. Through histogram equalization and fast median filtering algorithm, the plate image is smoothed and the noise in the image is filtered.

Edge detection of Sobel operator. The image is convolution from left to right, top to down by using the Sobel operator template, get the image plate edges information.

binary segmentation. The foreground color and background color of the edge image are obviously different, and the gray histogram of the image has obvious bimodal characteristics, find the binary image threshold in the histogram, and ultimately get a binary image containing only 0 and $1[6,7]$.

Boundary tracking. The object of boundary tracing is to remove the interference part, obtain the collection of only the plate boundary points. The search procedure of the boundary point sets use the stack method, and search the closed curve in the binary image. Through the area and the rectangle shape, remove the interference factor.

Detection of plate boundary line. Using Hough transform method to detect the points on the plate contour, the linear equation of the plate four sides can be obtained [8]. The rectangle of the four straight lines is the plate outline, then calculate the mean value of angle, as the plate angle, between two long sides and the shaft according to the four linear equation.

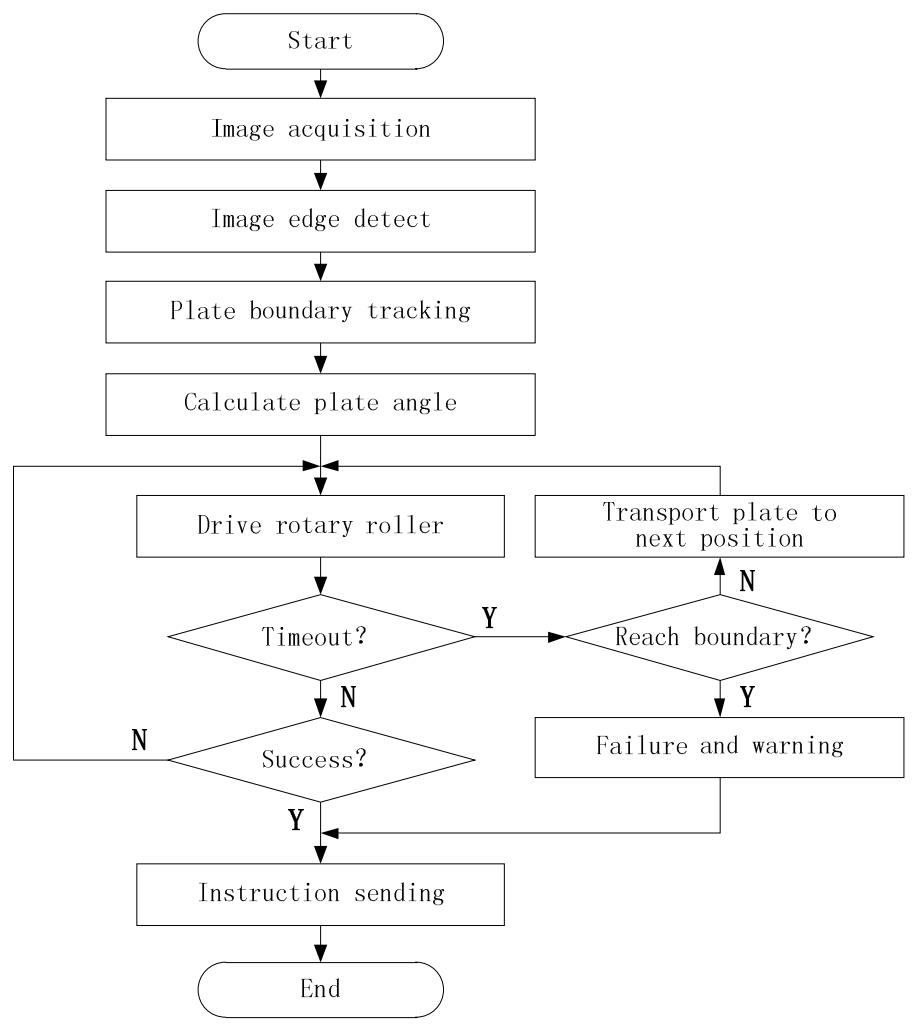

Fig. 3 Flow chart of turning plate control

When the plate is first transported to turning plate position, the control command is generated by the image processing computer, control tapered roller by the programmable controller, and perform a 
turning plate operation. The definition of the turning command generation and the judgment of Arrival position is as follows:

Determine of Turning plate in place. Through the plate two long side to calculate plate angle $\alpha$ satisfy the following: $90-\theta<\alpha<90+\theta$ ( $\theta$ is critical value of angle error for turning plate), then confirm the plate to reach the end location.

Turning plate overtime. In the process of turning plate, due to the plate warp, down bend, slip or other factors, make the duration of turning plate $t$ to meet the following: $t>t_{0}\left(t_{0}\right.$ is a critical value for the duration of turning plate), then judge turning plate timeout.

Turning plate overtime processing. If the plate turn plate timeout at a certain position, then stop turning, send command to transport the plate to the next position, continue to turn plate. If all the positions of the turning plate were out of time, the failure information of turning plate will be generated, which is transmitted to the programmable controller. The control flow chart of turning plate is shown in Fig. 3.

\section{Measurement and Application}

Based on the above image processing algorithm, the plate angle in image was measured, and the online feedback value of the plate angel is obtained. Plate boundary identification process is shown in figure 4.

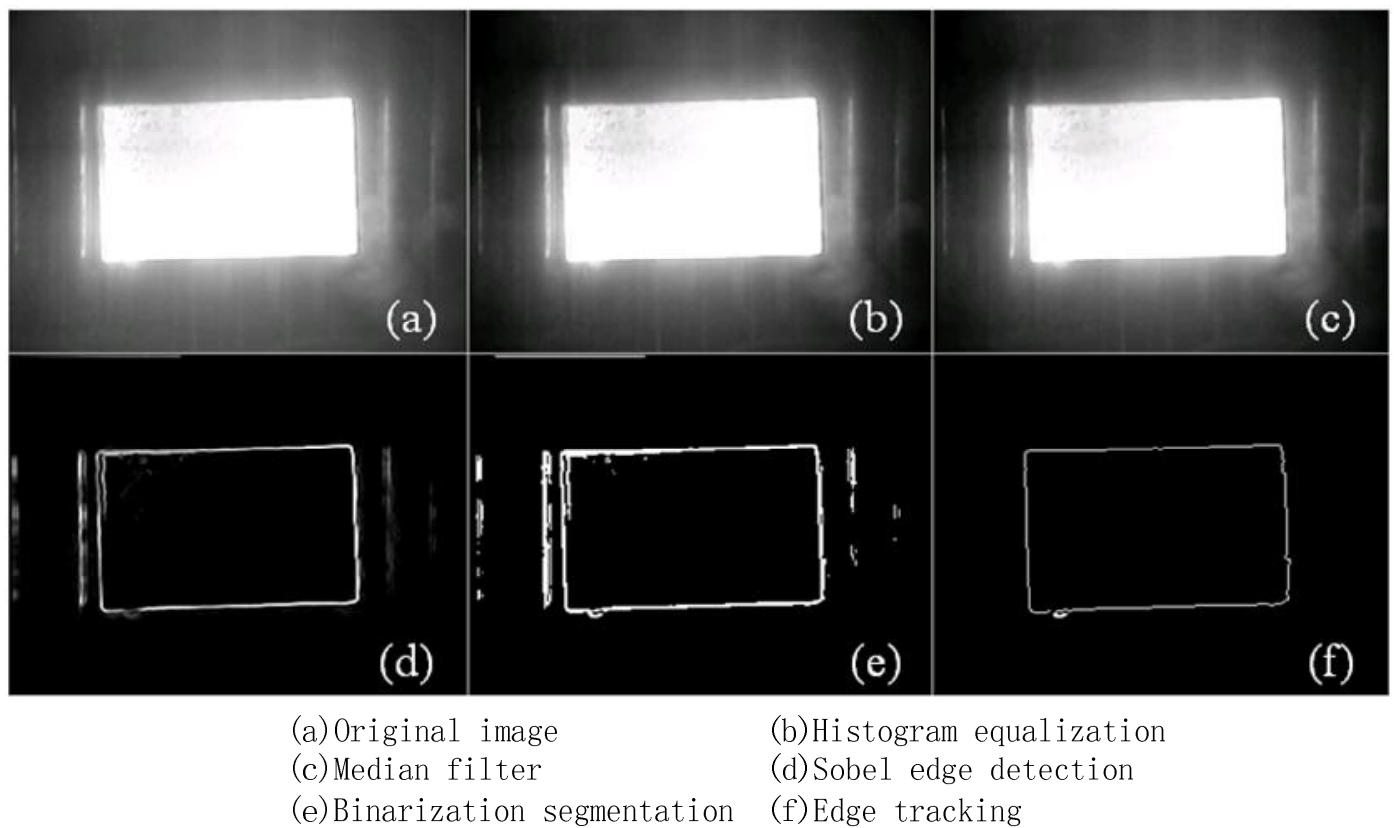

Fig. 4 The identification process of plate boundary

Plate image is processed by image processing algorithm, including edge detection, two value segmentation, boundary tracking, and Hough transform. The lines of the four sides of the plate are obtained, and the linear parameters are shown in Table 1. The obtained line and the original image are synthesized, the four sides line and the actual edge of the plate are in good agreement. The paper shows that the algorithm can meet the requirements of turning plate angel control

Table 1 The detection result of linear parameter

\begin{tabular}{ccc}
\hline Plate side & $\rho$ & $\theta$ \\
\hline 1 & -169 & -1.54 \\
2 & -62 & -1.55 \\
3 & 67 & 0.02 \\
4 & 247 & 0.04 \\
\hline
\end{tabular}


In order to realize the automatic control of the plate angle, the image processing computer needs to transmit the measurement to the control system. The computer and the control system adopts Ethernet communication, the communication interface adopts two ways, one is the communication between the OPC technology and the man-machine interface, which displays the measurement and control data of the plate rotation angle; the other is to communicate directly with the programmable controller through the TCP/IP interface. Communication mode as shown in Fig. 5.

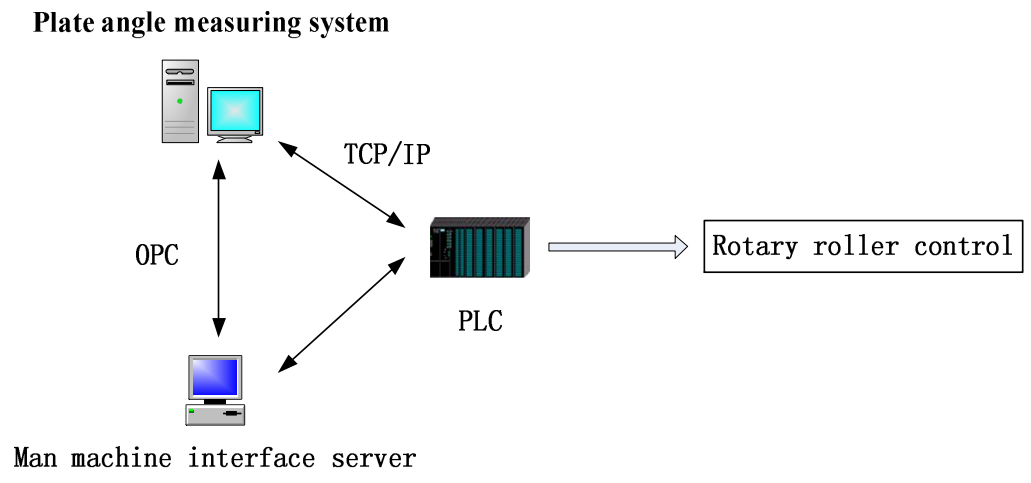

Fig. 5 Communication way of computer

After the production process testing, the plate angle measurement algorithm is fast, and the algorithm is less than $100 \mathrm{~ms}$, which meets the requirement of on-line measurement and control. According to the above discussion of the plate control logic to judge, the image processing computer measures the plate angle, and transfers control instruction to the programmable controller based on the communication interface, which realizes the on-line control of the plate angle.

\section{Conclusion}

According to the image processing method of gray histogram equalization, fast median filtering, edge detection, Hough transform line detection, this article develops a boundary tracking algorithm, which can automatically remove the interference, obtain boundary point set, realize the rapid measurement of the plate rotation angle.

Based on the requirement analysis of the plate rotation control, the angle control method is put forward, and the hardware and software structure of the system are designed. On the basis of considering various interference factors, the on-line control system of the turning plate is developed, and the application of the full automatic rolling is realized. The development of the automatic turning plate function meets the requirements of the production rhythm control. It has important practical significance for the uniform control of the high level product performance.

\section{References}

[1] H. Mitsunori, F. Nobuchika. Mitsubishi Electric ADVANCE Vol. 6(1997), p. 15

[2] T.M. Murphy, R.L. Johns: Iron and Steel Engineer Vol. 70(1993), p. 23

[3] A.J. Tabatai, O.R. Mitchell: IEEE Trans.Pattern Analysis and Machine Intelligence Vol. 6(1984), p. 188

[4] R.J. Montague, J. Watton, K.J. Brown. Materials Processing Technology Vol. 168(2005), p. 172

[5] T. Shiraishi, H. Ibata: ISIJ International Vol. 31(1991), p. 583

[6] F. Lahajnar, S. Kovacic: International journal of advanced manufacturing technology. Vol. 21(2003), p. 452

[7] K. David, P. James and G. Robert: The International Journal of Advanced Manufacturing Technology Vol. 28(2006), p. 781 
[8] W. Li, X.H. Huang, M. Wang. Signal Process Vol. 15(1999), p. 182 